Wei Yongqiao

Yang Shuhong

Zhang Qi $\bowtie$

Wang Yulin

Hou $\mathrm{Li}$

https://doi.org/10.21278/TOF.42106

ISSN 1333-1124

eISSN 1849-1391

\title{
NUMERICAL ANALYSIS OF ISOTHERMAL \\ ELASTOHYDRODYNAMIC LUBRICATION OF CYLINDRICAL GEARS WITH VARIABLE HYPERBOLIC CIRCULAR ARC AND TOOTH TRACE
}

\begin{abstract}
Summary
In this study on cylindrical gears with variable hyperbolic circular arc and tooth trace (VH-CATT), the tooth surface equation of the VH-CATT gear is obtained according to the theory of meshing and differential geometry. The data points of the tooth surface are obtained using MATLAB. Then, based on the data points, a model of a VH-CATT gear pair was built using UG. Considering the geometric features, contact form, relative velocity, entrainment velocity, load, etc., an isothermal elastohydrodynamic lubrication model of a VH-CATT gear pair is established. On the basis of the existing elastohydrodynamic lubrication (EHL) theory, an isothermal EHL numerical solution of a VH-CATT gear is obtained by utilizing the multigrid method. The distribution of dimensionless pressure and film thickness under the effect of various loads and at different speeds of a VH-CATT gear pair are discussed. The results show that both loading and speed have impact on oil film pressure and oil film thickness. The second pressure peaks and the minimum film thickness of the VH-CATT gear pair are greatly affected by the entrainment velocity and load. Based on the results of the study, we can provide a guide to creating a tribological design of VH-CATT gears and provide a theoretical basis and the application of value engineering in industrial applications of VH-CATT gear pairs under high speed and heavy load.
\end{abstract}

Key words: $\quad$ VH-CATT gear; entrainment velocity; numerical calculation; oil film pressure; oil film thickness

\section{Introduction}

With the rapid development of modern industry and the evolution to "Industry 4.0", gears are required to have a greater load bearing capacity and higher transmission efficiency in engineering fields of aerospace, construction machinery, mining machinery and so on, which leads to many development opportunities for the new gear [1]. Studies dealing with 
gears have been carried out by many researchers. The VH-CATT gear is a new type of gears which combines the characteristics of a traditional involute spur gear, a helical cylindrical gear and a herringbone gear. The VH-CATT gear has an arc contact line along the direction of the tooth line and the arc tooth surface. In comparison with the involute spur gear and the helical gear, the VH-CATT gear has the arc tooth line as well as better meshing performance, such as higher contact strength, larger load bearing capacity, higher contact ratio and no axial force. Therefore, the VH-CATT gear can better adapt to requirements in the field of aviation and aerospace in which transmissions require the gear used at high speed and heavy load. In addition, the VH-CATT gear has also other characteristics, such as smooth transmission, low noise, long life and easy installation. Based on these favourable features, such gears have good application prospects [2-4].

The VH-CATT gear is derived from the curved tooth bevel gear. At the beginning of the twentieth century, more and more researchers began to attach importance to the curved bevel gear and the Gleason Corp was the first to develop the circular arc tooth line bevel gear [5]. Because of the complex meshing characteristics and manufacturing process of VH-CATT gears, the VH-CATT gear is susceptible to failures of pitting, gluing, and wear caused by poor lubrication in the transmission process. From the literature [6,7], it is known that currently there is not much research going on in the lubrication performance of VH-CATT gears. Therefore, to improve their transmission lubrication properties and their application in the fields of aviation and aerospace it is very important to conduct in-depth studies on lubrication parameters and lubrication conditions of VH-CATT gears.

In 1916, Martin applied the Reynolds equation to the research into gear lubrication for the first time. Gears were simplified as two cylindrical rollers, and the change regularity of pressure distribution and film thickness with velocity and load was obtained, yet it was quite far from being practical [8]. In 1950s, Dowson and Higginson produced a full numerical solution of the line contact lubrication model using the inverse method [9]. The EHL theory which considers contact deformation at the interface in [10] provides a basis for the study on gear lubrication. The thermal EHL of helical gears was analyzed by means of the finite difference method [11], and based on the calculation result, the formula of load capacity of the gear, the maximum oil film temperature and the coefficient formula were fitted regressively. Spur gear thermal EHL was discussed in [12] with respect to the multigrid method. Liu [13] established an analysis model of the finite length thermal EHL for the helical gear and discussed the change rule of oil film pressure, thickness and temperature. Thermal EHL of helical gears, hypoid gears and worm gears were analyzed in [14], in which the effect of rotational speed on load capacity, oil film pressure and temperature was obtained. V. Simon established a thermal EHL analysis model of a hypoid gear, based on its characteristics [15]. Wang Jiaxu and others have conducted a study on interfacial lubrication of a hypoid gear under heavy load [16]. Dealing with EHL and thermal EHL of spur, bevel and hypoid cylindrical gears, Hou Li established an EHL model of VH-CATT gears and the effect of load, velocity, and viscosity on oil film pressure and thickness were also discussed [17]. However, there is no published study on the analysis of thermal EHL of VH-CATT gears.

In this paper, based on the principle of meshing of VH-CATT gears and the EHL theory, the equation of the tooth surface of VH-CATT gear is established, and the tooth flank curvature and relative velocity (entrainment velocity) of the meshing surface is calculated. The effect of load and entrainment velocity on the oil film pressure and thickness are also discussed. The result of the study can be seen as a guide to creating the tribological design of VH-CATT gears. 


\section{Equation of tooth surface}

The method of forming is machining cylindrical gears with an arcuate tooth trace with a rotary cutter head. In such a method, the rotary cutter head rotates and the gear blank rotates and moves along the rotary cutter head. The position, the coordinate system and the coordinate transformation of the rotary cutter head and gears are shown in Figure 1. The $O-X, Y, Z$ axes make the static coordinate system, where $\vec{i}, \vec{j}, \vec{k}$ are the coordinate basis. The $O_{1}-X_{1}, Y_{1}, Z_{1}$ axes make the coordinate system which is fixed by the rotary cutter head, where $\overrightarrow{i_{1}}, \overrightarrow{j_{1}}, \overrightarrow{k_{1}}$ are the coordinate basis. The $O_{2}-X_{2}, Y_{2}, Z_{2}$ axes make the coordinate system which is fixed by the gear blank, where $\vec{i}_{2}, \overrightarrow{j_{2}}, \overrightarrow{k_{2}}$ are the coordinate basis. $R_{1}$ is the pitch radius of the gear blank. $R_{T}$ is the nominal cutter radius.

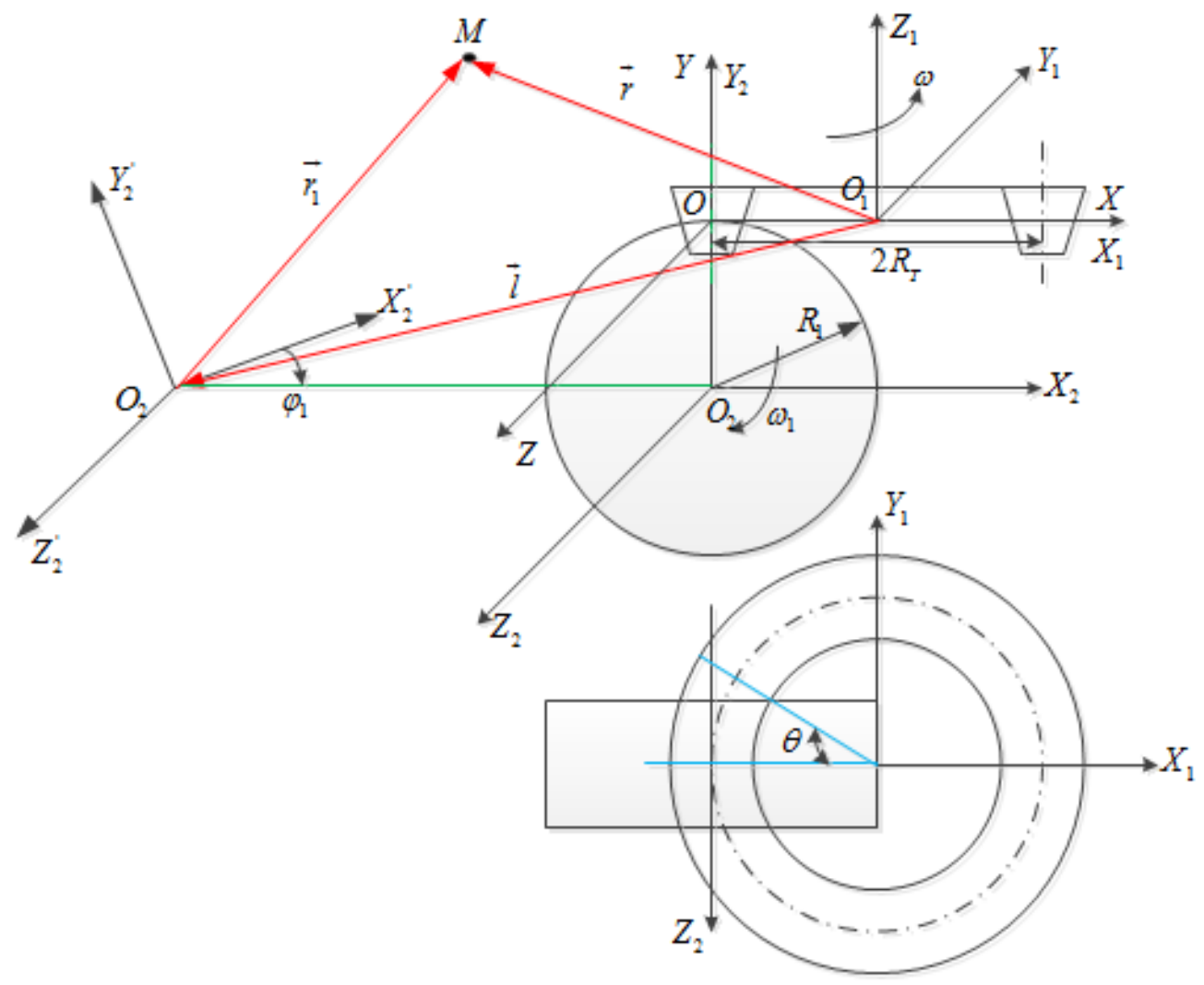

Fig. 1 Principle of forming a VH-CATT gear

In the coordinate system of the rotary cutter, the parameter equation of the cutter surface is given as follows:

$$
\Sigma_{1}:\left\{\begin{array}{c}
x_{1}=-\left(R_{T} \pm \pi m / 4 \pm u \sin \alpha\right) \cos \theta \\
y_{1}=\left(R_{T} \pm \pi m / 4 \pm u \sin \alpha\right) \sin \theta \\
z_{1}=u \cos \alpha
\end{array}\right.
$$

where $u$ is the distance in the cutter coordinate system from the point on the cutting surface to the envelop reference point along the cutting cone generatrix, $\alpha$ is the pressure angle of tool (equal to the cone semi-angle), $\theta$ is the angle through which the gear blank is rotated from the middle section to the current enveloping point. When $\theta$ equals zero the reference point is in the middle section. 
The gear angle of rotation $\varphi=\omega_{1} \cdot t$ is the motion parameter, by which the tool surface parameter equation could be transformed to the gear blank coordinate system and the tooth surface equation $\Sigma_{2}$ is established. The transformation matrix $M_{21}$ is transformed from $O_{1}-x_{1}, y_{1}, z_{1}$ to $O_{2}-x_{2}, y_{2}, z_{2} . M_{21}$ is given as follows:

$$
\begin{aligned}
M_{21} & =\left[\begin{array}{cccc}
\cos \varphi_{2} & \sin \varphi_{2} & 0 & 0 \\
-\sin \varphi_{2} & \cos \varphi_{2} & 0 & 0 \\
0 & 0 & 1 & 0 \\
0 & 0 & 0 & 1
\end{array}\right]\left[\begin{array}{cccc}
1 & 0 & 0 & R_{1} \varphi_{2} \\
0 & 1 & 0 & 0 \\
0 & 0 & 1 & 0 \\
0 & 0 & 0 & 1
\end{array}\right]\left[\begin{array}{cccc}
1 & 0 & 0 & R_{T} \\
0 & 0 & 1 & R_{1} \\
0 & -1 & 0 & 0 \\
0 & 0 & 0 & 1
\end{array}\right] \\
\left\{\sum_{2}\right\} & :\left[\begin{array}{c}
x_{2} \\
y_{2} \\
z_{2} \\
1
\end{array}\right]=M_{21}\left[\begin{array}{c}
x_{1} \\
y_{1} \\
z_{1} \\
1
\end{array}\right]
\end{aligned}
$$

The tooth surface equation of the VH-CATT is obtained according to the cutting tool and the tooth surface envelope.

$$
\left\{\begin{array}{l}
x_{2}^{\prime}=\left[-\left(R_{T} \pm \pi m / 4 \pm u \sin \alpha\right) \cos \theta+R \varphi+R_{T}\right] \cos \varphi-(u \cos \alpha+R) \sin \varphi \\
y_{2}^{\prime}=\left[-\left(R_{T} \pm \pi m / 4 \pm u \sin \alpha\right) \cos \theta+R \varphi+R_{T}\right] \sin \varphi+(u \cos \alpha+R) \cos \varphi \\
z_{2}^{\prime}=-y_{1}=-\left(R_{T} \pm \pi m / 4 \pm u \sin \alpha\right) \sin \theta \\
u=\mp \sin \alpha\left(R_{T} \mp \pi m / 4\right)+\left(R \varphi+R_{T}\right) \sin \alpha / \cos \theta
\end{array}\right.
$$

\section{Meshing model}

The point cloud of the tooth surface of the VH-CATT is generated in MATLAB on the basis of the principle of meshing and tooth surface equation, as shown in Fig. 2(a). The tooth surface is formed from the point cloud using the curved surface fitting function in NX 10.0. The ideally instantaneous contact line of the gear is the space line, the gear has an arc curve in the tooth line direction and an involute in the tooth profile direction. The model of the meshing of the VH-CATT gear is shown in Fig. 2(b). The type of contact of the VH-CATT gear is the point contact, and the size of the contact zone is determined by the contact ellipse. The contact ellipse is shown in Fig. 3.

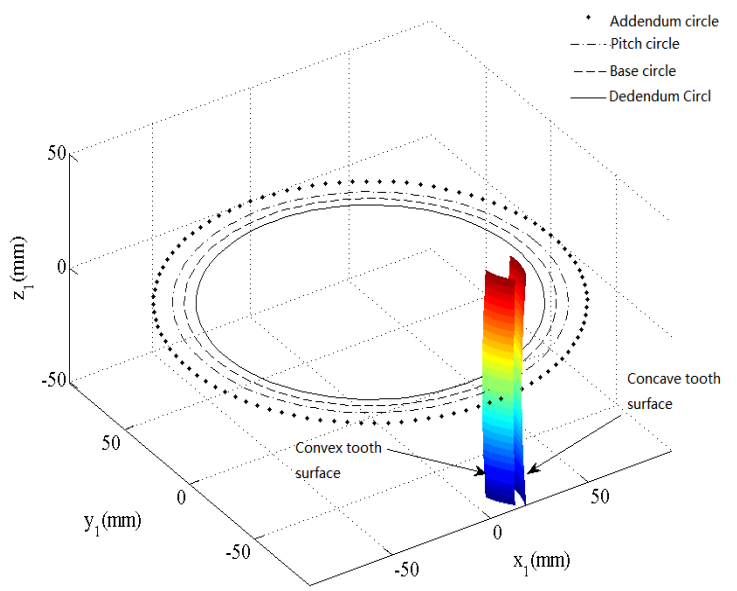

(a)

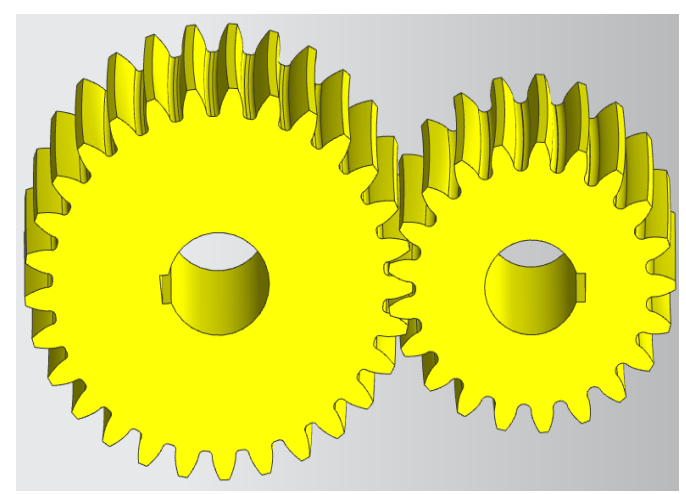

(b)

Fig. 2 Meshing model of the VH-CATT gear 
Numerical Analysis of Isothermal

Elastohydrodynamic Lubrication of Cylindrical Gears with

Variable Hyperbolic Circular Arc and Tooth Trace
W. Yongqiao, Y. Shuhong, Z. Qi, W. Yulin, H. Li
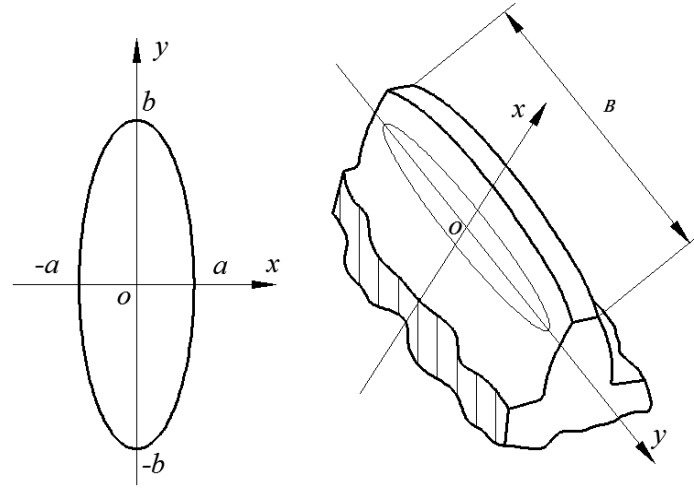

Fig. 3 Model of the contact ellipse

According to the tooth surface equation, VH-CATT gears are manufactured with a CNC machine tool; the manufacture of the sample is shown in Fig. 4. The gear pair exhibits the meshing properties and geometric features with respect to applied loads, whereby the correctness of the tooth surface equation is verified.

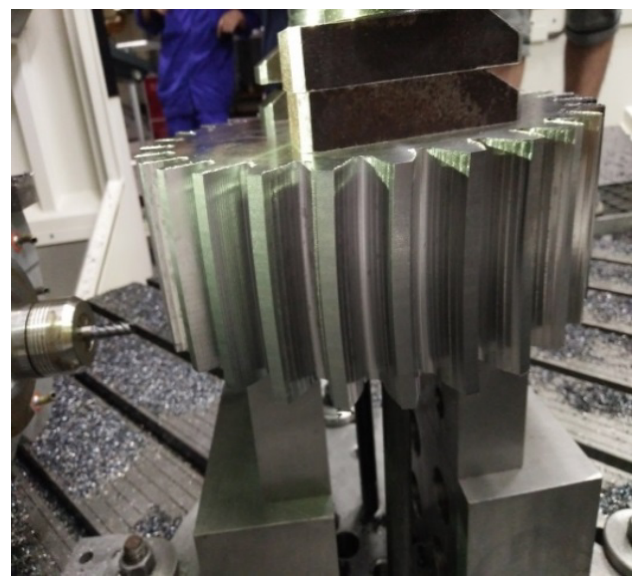

(a)

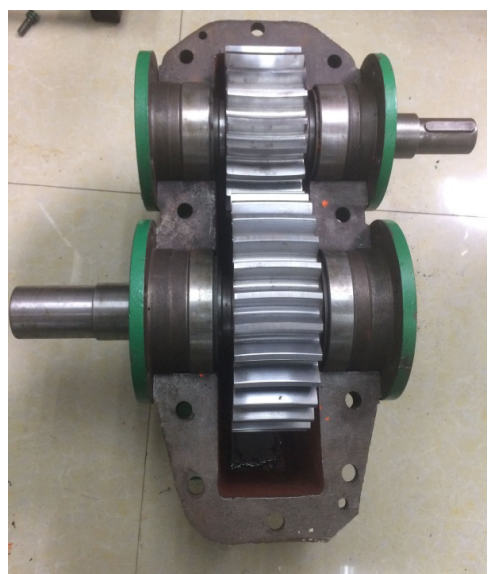

(b)

Fig. 4 Manufacture of the VH-CATT gear sample

The meshing transmission diagram of the VH-CATT gear is shown in Fig. 5, where two gears rotate in the opposite direction, $K$ is the space meshing point, $a$ is the centre distance. $w_{1}$ and $w_{2}$ are gear angular velocities.

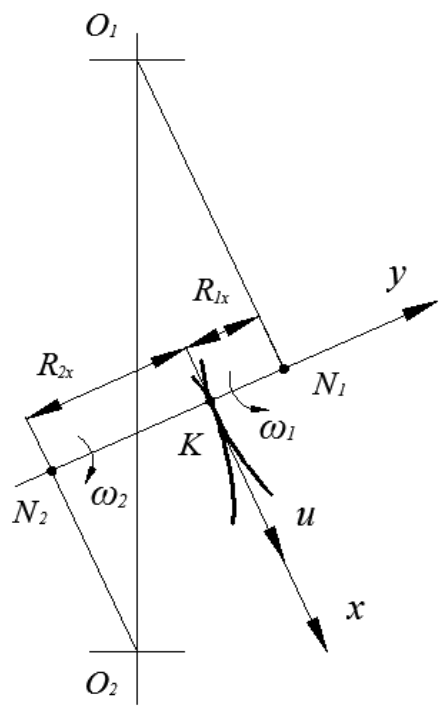

Fig. 5 The relationship between geometry and kinematics of the VH-CATT gear 
Based on the elastohydrodynamic lubrication (EHL) theory, the velocities of two tooth surfaces of the VH-CATT gear at the meshing point are given as follows:

$$
\begin{aligned}
& \mu_{1}=R_{1 x} \omega_{1}=\frac{\pi n_{1}}{30 K_{2}^{I}} \\
& \mu_{2}=R_{2 x} \omega_{2}=\frac{\pi n_{2}}{30 K_{2}^{I I}}
\end{aligned}
$$

According to [18], the entrainment velocity of the VH-CATT gear at the meshing point can be obtained as follows:

$$
\mu=\frac{\mu_{1}+\mu_{2}}{2}=\frac{\pi}{60}\left(\frac{n_{1}}{K_{2}^{I}}+\frac{n_{2}}{K_{2}^{I I}}\right)
$$

\section{Lubrication model}

The Reynolds equation of isothermal EHL of the point contact is expressed as follows $[19,20]$ :

$$
\frac{\partial}{\partial x}\left(\frac{\rho h^{3}}{\eta} \frac{\partial p}{\partial x}\right)+\frac{\partial}{\partial x}\left(\frac{\rho h^{3}}{\eta} \frac{\partial p}{\partial y}\right)=12 \mu \frac{\partial(\rho h)}{\partial x}
$$

where $p$ is the film pressure, $h$ is the film thickness, $\rho$ is the density of lubrication oil, $\eta$ is the viscosity of lubrication oil, $u$ is the entrainment velocity.

The formula ( 8 ) is normalized by the introduced dimensionless parameters.

$$
\begin{aligned}
& X=x / a, Y=y / b, P=p / p_{h}, \bar{\rho}=\rho / \rho_{0}, \bar{\eta}=\eta / \eta_{0}, H=h R_{x} / a^{2}, G=\alpha E, \\
& U=\eta_{0} u / E R_{x}, \bar{F}=F / E R_{x}^{2}, e_{k}=R_{x} / R_{y}, \frac{1}{E^{\prime}}=\frac{1}{2}\left(\frac{1-v_{1}^{2}}{E_{1}}+\frac{1-v_{2}^{2}}{E_{2}}\right)
\end{aligned}
$$

where $a$ and $b$ are the contact half-width, $\eta_{0}$ is the initial viscosity, $\rho_{0}$ is the initial density, $p_{h}$ is the maximum Hertzian contact pressure, $E^{\prime}$ is the synthetic modulus of elasticity of gear pairs, $E_{1}, E_{2}$ and $v_{1}, v_{2}$ are the modulus of elasticity and Poisson's ratio of the gears, respectively.

The normalized equation (8) is given as follows :

$$
\frac{\partial}{\partial X}\left(\varepsilon_{X} \frac{\partial P}{\partial X}\right)+\frac{\partial}{\partial Y}\left(\varepsilon_{Y} \frac{\partial P}{\partial Y}\right)=\frac{\partial(\bar{\rho} H)}{\partial X}
$$

where

$$
\begin{aligned}
& P\left(X_{\text {in }}, Y\right)=P\left(X_{\text {out }}, Y\right)=P\left(X, Y_{\text {in }}\right)=P\left(X, Y_{\text {out }}\right)=0, \frac{\partial P}{\partial X}=\frac{\partial P}{\partial Y}=0, \\
& \varepsilon_{X}=\frac{a p_{h}}{12 \eta_{0} \mu}\left(\frac{\bar{\rho} H^{3}}{\bar{\eta}}\right), \quad \varepsilon_{Y}=\frac{a^{3} p_{h}}{12 b^{2} \eta_{0} \mu}\left(\frac{\bar{\rho} H^{3}}{\bar{\eta}}\right), X_{\text {in }}<X<X_{\text {out }}, Y_{\text {in }}<Y<Y_{\text {out }}
\end{aligned}
$$

The following formulae are rewritten according to the above normalization method. Due to the paper length limit, the original equations are omitted and the normalized equations are given. 
The normalized film thickness equation is given as follows :

$$
H(X, Y)=H_{0}+\frac{X^{2}+e_{k} Y^{2}}{2}+M \int_{X_{0}}^{X_{e}} \int_{Y_{0}}^{Y_{e}} \frac{P(S, T)}{\sqrt{(X-S)^{2}+(Y-T)^{2}}} \mathrm{~d} S \mathrm{~d} T
$$

where $H_{0}$ is the normalized center film thickness, $X_{0}, X_{e}, Y_{0}, Y_{e}$ are the normalized coordinates of the boundary, and $M$ equals $\frac{3 F R_{x}}{\pi^{2} a^{2} b E}$

The normalized density pressure equation is given as follows :

$$
\bar{\rho}=1+\frac{0.6 P \cdot p_{h}}{1+1.7 P \cdot p_{h}}
$$

The normalized viscous pressure equation is given as follows :

$$
\bar{\eta}=\exp \left\{\left(\ln \eta_{0}+9.67\right)\left[\left(1+P \frac{p_{h}}{p_{0}}\right)^{z}-1\right]\right\}
$$

The normalized loading equation is given as follows :

$$
\int_{X_{0}}^{X_{e}} \int_{Y_{0}}^{Y_{e}} P(X, Y) d X d Y=\frac{2 \pi}{3}
$$

For this study of numerical solutions of the point contact EHL, the differential method is used for developing a lubrication model. The above equations are discretized by using the central difference and forward and backward differences. The normalized Reynolds equation is discretized as follows:

$$
\frac{\varepsilon_{i-1 / 2, j} P_{i-1, j}+\varepsilon_{i+1 / 2, j} P_{i+1, j}+\varepsilon_{i, j-1 / 2} P_{i, j-1}+\varepsilon_{i, j+1 / 2} P_{i, j+1}-\varepsilon_{0} P_{i, j}}{\Delta X^{2}}=\frac{\bar{\rho}_{i, j} H_{i, j}-\bar{\rho}_{i-1, j} H_{i-1, j}}{\Delta X}
$$

where

$$
\varepsilon_{i \pm 1 / 2, j}=\frac{1}{2}\left(\varepsilon_{i, j}+\varepsilon_{i \pm 1, j}\right) ; \varepsilon_{0}=\varepsilon_{i-1 / 2, j}+\varepsilon_{i+1 / 2, j}+\varepsilon_{i, j-1 / 2}+\varepsilon_{i, j+1 / 2}
$$

The discretized film thickness equation is given as follows:

$$
H_{i, j}=H_{0}+\frac{X_{i}^{2}+e_{k} Y_{j}^{2}}{2}+M \sum_{k=1}^{n} \sum_{l=1}^{n} D_{i j}^{k l} P_{k l}
$$

where $D_{i j}^{k l}$ is the two-dimensional elastic stiffness coefficient.

The discretized loading equation is given as follows:

$$
W=\Delta X \Delta Y \sum_{i=1}^{n} \sum_{j=1}^{n} P_{i j}=\frac{2 \pi}{3}
$$




\section{Numerical calculation}

Based on the established mathematical model, the calculation flow chart shown in Fig. 6 is used for the iterative solution. The calculation parameters of the gear pair are given in Table 1.

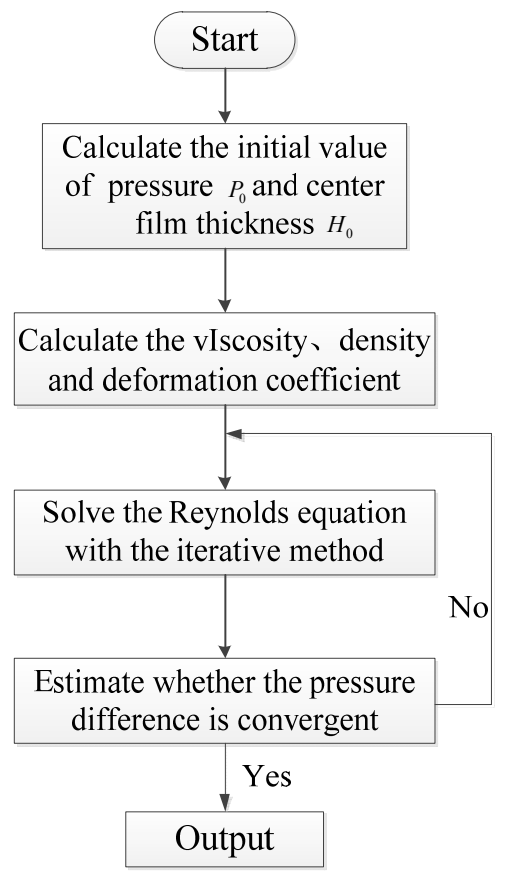

Fig. 6 Calculation flow chart

Table 1 Parameters of the gear pair

\begin{tabular}{ccc}
\hline Parameter & Driving wheel & Driven wheel \\
\hline Tooth Number & 21 & 29 \\
Modulus & 8 & 8 \\
Tooth width $/ \mathrm{mm}$ & 84 & 80 \\
Pressure angle & 20 & 20 \\
Tooth line radius $/ \mathrm{mm}$ & 500 & 500 \\
\hline
\end{tabular}

The modulus of elasticity of the gear material is $2.21 \times 10^{11} \mathrm{~Pa}$; Poisson's ratio is 0.28 ; the initial viscosity of the lubricating oil is $0.02 \mathrm{~Pa} \cdot \mathrm{s}$. The environmental temperature is set to $25^{\circ} \mathrm{C}$, the viscosity-pressure coefficient is set to $2.2 \times 10^{-8} \mathrm{~m}^{2} / \mathrm{N}$. The entrainment velocity can be calculated according to formula (7). According to the gear EHL model and applying the iterative calculation with the above mentioned parameters, distributions of the oil film pressure and the film thickness shape of the VH-CATT gear are obtained.

Characteristic parameters of the lubrication characteristics of the VH-CATT gear are mainly EHL oil film pressure and oil film thickness. It can be seen that the characteristic parameters of the effect were mainly entrainment speed, load, and the modulus of elasticity of the material as well as viscosity of lube base oil. The impact of entrainment velocity and load on the oil film pressure and the oil film thickness of the VH-CATT gear will be discussed in the next subsections. 


\subsection{Effect of load on oil film pressure and oil film thickness}

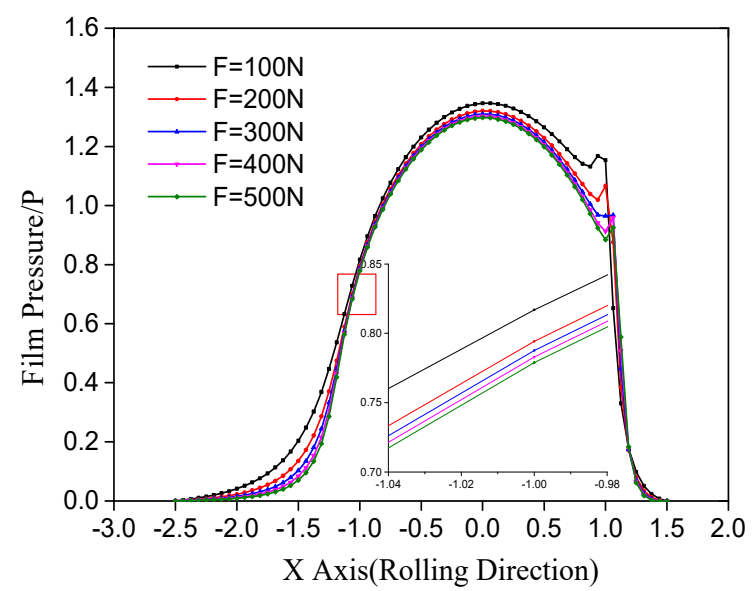

(a)

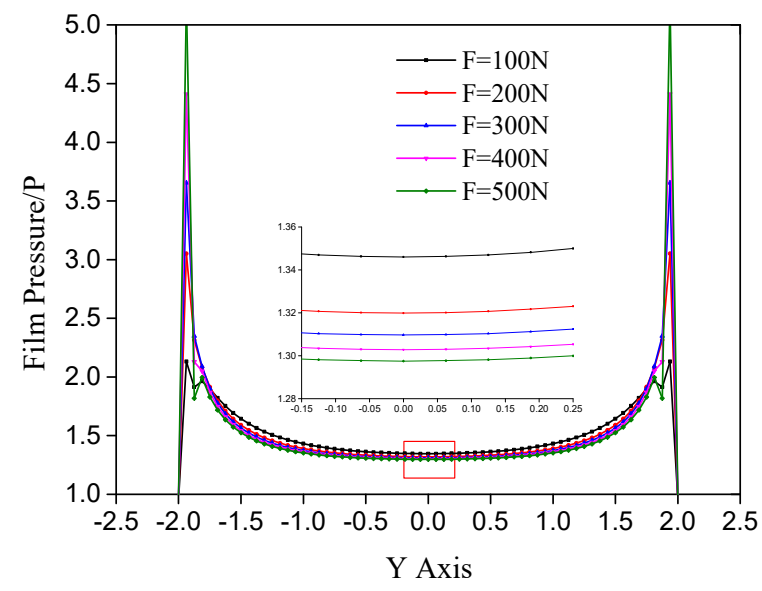

(c)

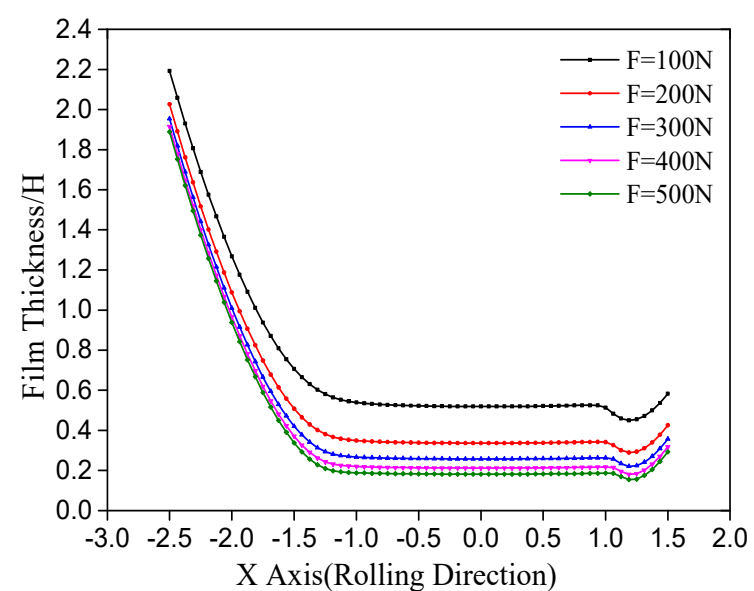

(b)

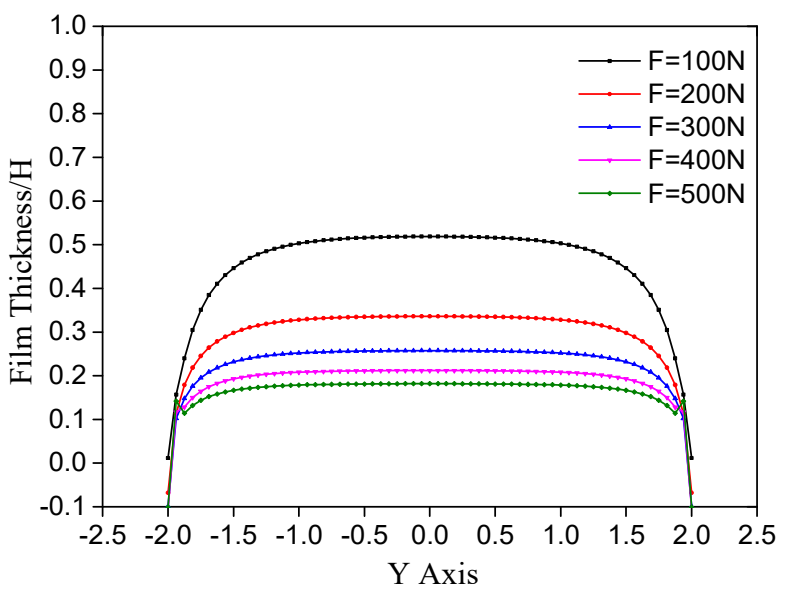

(d)

Fig. 7 Effect of load on the distribution of oil film pressure and film thickness

Fig. 7 shows the Effect of load on the distribution of oil film pressure and film thickness in the $\mathrm{X}$ axis (rolling direction) and the $\mathrm{Y}$ axis. It could be seen from Fig. 7(a) that the distribution of oil film pressure is close to the Hertz pressure distribution in the rolling direction. Oil film pressure reaches its second peak near the exit zone, but the second pressure decreases rapidly to the value of the ambient pressure. It is shown that the second pressure peak of the oil film decreases slowly with an increase in load, and the pressure position moves to the entrance zone. It could be seen from Fig.7 (b) that the neck-in phenomenon of film thickness occurs in the vicinity of the second pressure peak, and the film thickness reaches its minimum at the neck-in phenomenon position in the rolling direction. It could be seen from Fig. 7(c) and (d) that the film thickness reaches its maximum at the centre zone and its minimum on the two sides in the $\mathrm{Y}$ axis (tooth line direction). With an increase in load, the film thickness and film pressure decrease, the pressure changes on both sides, and the vibration phenomenon will appear with an increase in load. 
5.2 Effect of entrainment velocity on oil film pressure and oil film thickness

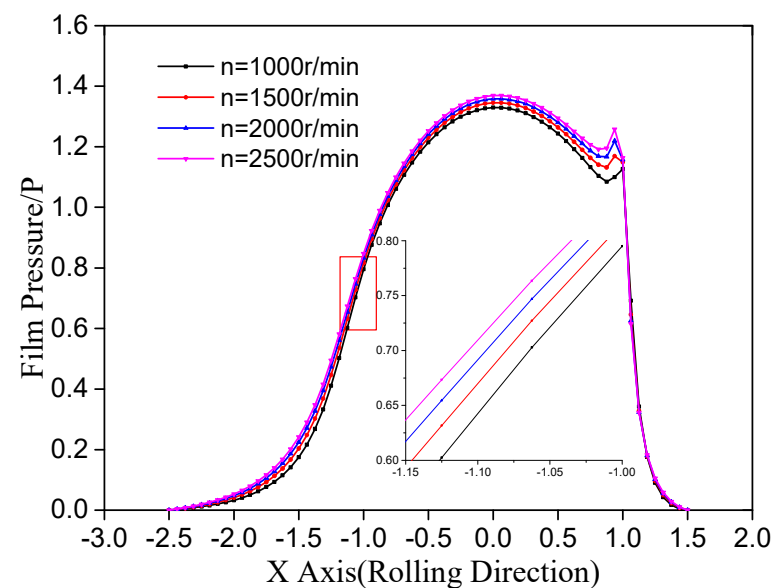

(a)

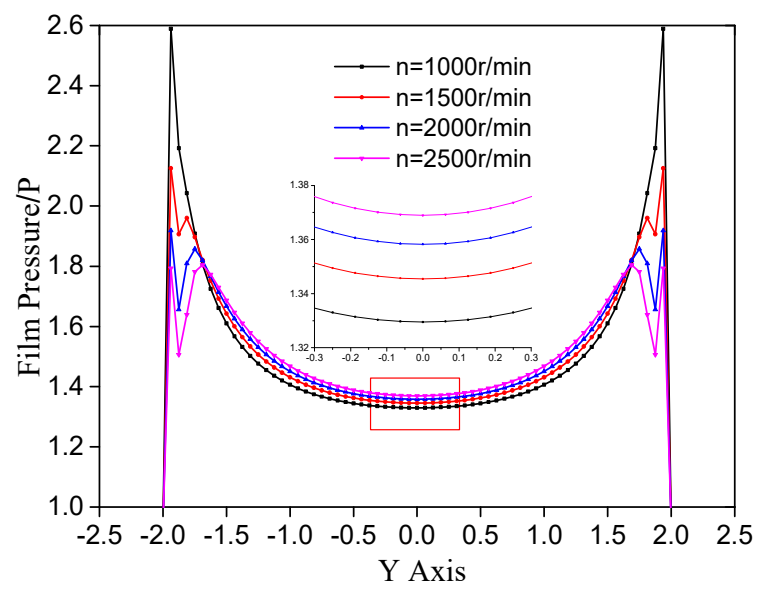

(c)

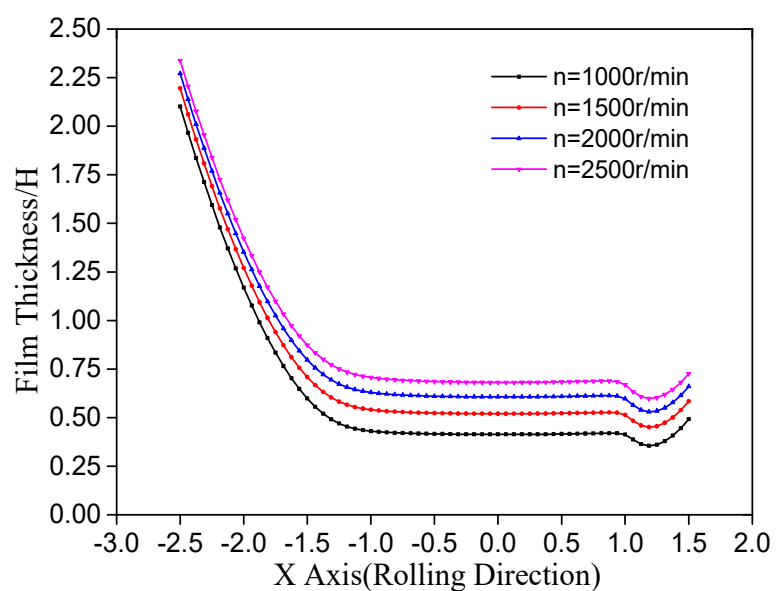

(b)

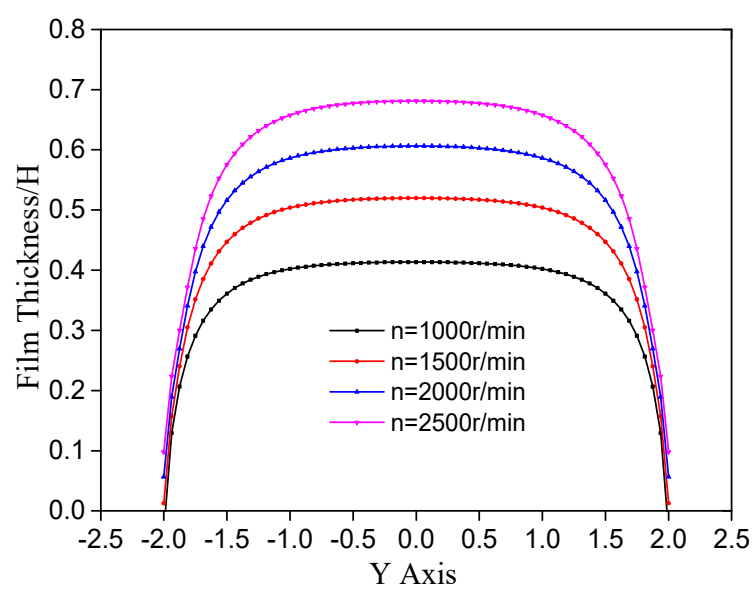

(d)

Fig. 8 Effect of entrainment velocity on the distribution of oil film pressure and film thickness

Fig. 8 shows the effect of entrainment velocity on the distribution of oil film pressure and film thickness in the $\mathrm{X}$ axis (rolling direction) and the $\mathrm{Y}$ axis. It could be seen from Fig. 8 that the distributions of oil film pressure and film thickness are the same as in Fig. 7 in the $\mathrm{X}$ axis (rolling direction). It could be seen from Fig. 8(a) and (c) that the oil film pressure increases with an increase in speed. The second pressure peak of the oil film increases with an increase in speed, and the pressure position moves to the entrance zone. But the oil film pressure changes at the two sides decrease with an increase in speed. It could be seen from Fig. 8(b) and (d) that the film thickness increases with an increase in entrainment velocity. The neck-in phenomenon of film thickness corresponding to the second pressure peak also occurs ahead of schedule and moves towards the direction of the entrance zone. So, the reasonable selection of the operating speed and load on gears is very important if the occurrence of scuffing on gear surfaces is to be avoided.

\section{Conclusions}

1) Based on the principles of meshing and machining of a VH-CATT gear, the tooth surface equation of the VH-CATT has been derived. Considering the contact characteristics, relative velocity, entrainment velocity, load and other factors synthetically, a mathematical model of the isothermal EHL for VH-CATT gears has been established. 
2) Employing the numerical method as the basis of calculation, the effect of entrainment velocity and load on oil film pressure and oil film thickness of VH-CATT gears has been discussed. It could be concluded that the oil film pressure and the oil film thickness decrease with an increase in load, but they increase with an increase in speed. And the greater the change in velocity and load is, the more likely the tooth surface scuffs. So, in order to avoid possible scuffing of gears as much as possible, the operating velocity and load of gears should be chosen adequately to make the lubrication of VH-CATT gears close to full EHL and ensure the best lubrication properties.

\section{Acknowledgment}

This project is supported by the National Natural Science Foundation of China (51375320) and the Panzhihua City Application and Development Foundation (2016CY-G10,2013TX8).

\section{REFERENCES}

[1] XIAO Hua-jun, HOU Li, DONG Lu, JIANG Yi-qiang, WEI Yong-qiao. Mathematical Modeling of Rotary Cutter Arc Tooth Line of Cylindrical Gear Shaped by Origin Face of Rotary Cutter. Journal of Sichuan University, 2013, 45, ( 5): 171-175

[2] WANG Shao-jiang, HOU Li, DONG Lu, XIAO Hua-jun. Modeling and Strength Analysis of Cylindrical Gears with Curvilinear Shape Teeth for Manufacture. Journal of Sichuan University, 2012,44 (5):210-213

[3] Song Aiping. Involute arc cylindrical gear and its mesh characteristics. China Mechanical Engineering, 2006, 9(18):1888-1892.

[4] Di Y., Chen M. Generation principle of accurate tooth trace cylindrical gear. Journal of Harbin Bearing. 2006, 27(3):58-61.

[5] Zhang, Q., Hou, L., Tang, R., Wen, G. Method of Processing and an Analysis of Meshing and Contact of Circular Arc Tooth Trace Cylindrical Gears. Transactions of FAMENA, 2016, 40(4):11-24. https://doi.org/10.21278/TOF.40402

[6] Wu Xutang. The spatial meshing principle and application. Beijing: Mechanical Industry Press, 1982

[7] Rui-Tang Tseng, Chung-Biau Tsay. Contact characteristics of cylindrical gears with curvilinear shaped teeth. Mechanism and Machine Theory, 2004, 39(9):905-919.

https://doi.org/10.1016/j.mechmachtheory.2004.04.006

[8] Martin H.M. The lubrication of gear teeth. Engineering, 1916, 102:119-121

[9] Dowson D., Higginson G.R. A numerical solution to the elastohydrodynamic problem. Journal of Mechanical Engineering Science, 1959, 1(1):6-15.

https://doi.org/10.1243/JMES_JOUR_1959_001_004_02

[10] ZHU D, WANG Q. Elastohydrodynamic lubrication: a gateway to interfacial mechanics: review and prospect. ASME J Tribol, 2011, 133(4):41-51

[11] V. Simon. Thermo-EHD Analysis of Lubrication of helical gears. ASME, Journal of mechanisms, Transmissions, and Automation in Design, 1988, 110:330-336. https://doi.org/10.1115/1.3267466

[12] Wang Youqiang, Yang Peiran. Transient Thermoelastohydrodynamic Lubrication Analysis of an involute spur gear. Chinese Journal of Mechanical Engineering, 2004, 40(9):10-15. https://doi.org/10.3901/JME.2004.09.010

[13] YANG Ping, YANG Peiran. Theory of Thermal Elastohydrodynamic Lubrication for Helical gears, Chinese Journal of Mechanical Engineering, 2006, 42(10):43-48.

https://doi.org/10.3901/JME.2006.10.043

[14] V. Simon. EHD Lubrication of different Types of Gears. Technical Sessions Proceedings of CIST2008\&ITS -IFToMM, Beijing, China, 2008,46-47

[15] V. Simon. Effect of Machine tool setting Parameters on EHD Lubrication in Hypoid Gears, Mechanism and Machine Theory, 2009, 44:923-937. https://doi.org/10.1016/j.mechmachtheory.2008.06.005

[16] PU Wei, WANG Jiaxu. Analysis on Lubrication Performance of Hypoid Gears at Heavy Loads. Journal of xi'an Jiaotong University, 2015:49(11):55-61. 
[17] Zhou Bo, Hou Li, Wei Yongqiao, Li Bo: Analysis and Establishment of the Isothermal Elastohydrodynamic Lubrication Model of the Cylindrical Gear with Arcuate Tooth Trace. Journal of Mechanical Transmission, 2015, 39(3):18-22

[18] Wen Shizhu, Yang Peiran. Elastohydro-Dynamic Lubrication. Beijing: Tsinghua Press, 1992.

[19] Huang Ping. Numerical Calculation Methods of Elastohydrodynamic Lubrication. Beijing: Tsinghua Press, 2013.

[20] Liu Mingyong. Research on Thermal Finite Line Contact EHL for Helical Gears. Chongqing University, 2013

Submitted: $\quad$ 15.6.2017 Wei Yongqiao

Accepted: $\quad 27.9 .2017$

School of Mechanical \& Electronical Engineering, Lanzhou University of Technology, Lanzhou, 730050, China

School of Manufacturing Science and Engineering, Sichuan University, Chengdu 610065, China

Yang Shuhong

School of Life Science and Engineering, Lanzhou University of Technology, Lanzhou, 730050, China

Zhang Qi (corresponding author)

School of Mechanical Engineering, Panzhihua University, Panzhihua, 610039, China

Wang Yulin

Maintenance Department, Civil Aviation Flight University of China, Guanghan, 618307, China

Li Hou

School of Manufacturing Science and Engineering, Sichuan University, Chengdu 610065, China 